\title{
Systematic review on the current Public Health Challenges for Dengue Transmission Control and Prevention in the Philippines
}

\author{
Gamaliel Issamar S. De Vera ${ }^{\mathrm{a}, \mathrm{h}}$, Jose Moses G. Cachola ${ }^{\mathrm{b}, \mathrm{h}}$, Jana Clarissa C. Magsino ${ }^{\mathrm{c}, \mathrm{h}}$, \\ Bianca Angela M. Pambid, ${ }^{\text {d,h }}$, Juliana Regina T. Policar ${ }^{\mathrm{e}, \mathrm{h}}$, Janine Krystel S. San Pedro ${ }^{\text {f,h }}$, \\ Ronn Angelo A. Taguibao ${ }^{\mathrm{g}, \mathrm{h} *}$
}

${ }^{a}$ gsdevera@ ust.edu.ph, bjosemoses.cachola.pharma@ust.edu.ph, cjanaclarissa.magsino.pharma@ust.edu.ph, dbiancaangela.pambid.pharma@ust.edu.ph, ${ }^{\mathrm{e}}$ julianaregina.policar.pharma@ust.edu.ph, fjaninekrystel.sanpedro.pharma@ust.edu.ph, ${ }^{\mathrm{g}}$ ronnangelo.taguibao.pharma@ust.edu.ph

${ }^{\mathrm{h}}$ Department of Medical Technology, Faculty of Pharmacy, University of Santo Tomas, España, Manila Philippines

\begin{abstract}
The dengue virus is a flavivirus endemic in over 100 countries, in which Southeast Asian countries are greatly affected due to the evident lack in public health intervention and laboratory resources. In 2019, the Philippines was able to observe a sudden increase in dengue cases, declaring a state of epidemic. The government then proposed several measures to address the sudden rise of dengue, which showed a significant decrease in dengue deaths during the first half of 2020, but dengue still remains a cause of concern given the uncertainty of possible future epidemics. Therefore, a systematic review was conducted according to the PRISMA 2020 guidelines using journals and articles published between Jan 1, 2015 and Dec 31, 2020 from Science direct, PubMed, HERDIN, Western Pacific Surveillance and Response (WPSAR), and Taylor and Francis Journal under the search strings "Dengue in the Philippines" and "Dengue prevention and control in the Philippines". The risk of bias was assessed using phase 2 and 3 of the ROBIS tool. A total of 19 articles identifying factors that influence public health interventions were included. Vector elimination and control (e.g., 4s strategy) through community participation has been the main focus of the current dengue prevention programs in the country, but these programs are presented with limitations. Various challenges and recommendations from different literatures were identified, but all consistently show the need for accurate identification and representation of dengue prevalence and burden to avoid higher overall healthcare costs, better involvement of the government and healthcare providers in information dissemination, advocacies with emphasis on behavioral change programs, and further research and development of a cost-effective, efficient, and less complex diagnostic method. It was therefore concluded that understanding the prevention and control of dengue in the Philippines would involve an elaborate public health investigation and review of several different factors aside from its viral mechanisms and pathogenesis, and that several aspects originating from individual behavior and practices of people up until coordination of LGUs, communities, and National-level agencies including coordinated budget allocations must all be aligned to efficiently lessen the prevalence of dengue. Research limitations encountered included the exclusive use of electronic databases and free/accessible published articles that inhibited the review's capability to include "grey literature", as well as the inability to have a registered protocol due to time constraints. An expansion in the criteria, databases used, and type of literature, along with the further exploring of each individual factor identified is recommended for future research.
\end{abstract}

Keywords: Dengue virus; public health response; vector control; disease burden; vector surveillance; dengue laboratory diagnosis; dengue vaccine; climate change; community-based programs 


\section{Introduction}

Dengue virus (DENV) is a flavivirus transmitted by the Aedes mosquito, known to cause large epidemics particularly in economically disadvantaged, tropical and subtropical countries (Viennet et al., 2016). The World Health Organization (2020) has estimated that the virus is endemic in over 100 countries, putting $50 \%$ of the world, 3.6 billion people, at risk. Seventy percent (70\%) of this population is concentrated in the Asia Pacific Region, with the Philippines ranking fourth among the countries of ASEAN (Ng, 2011; Bravo et al., 2014). The country has continued to observe fluctuations in the number of dengue cases recorded annually, as periodic outbreaks and epidemiological trends occur every 3-5 years under the influence of patterns and epidemiological factors (Polwiang, 2020).

The surveillance of dengue in the Philippines, along with other diseases, is done through the National Epidemic Sentinel Surveillance System and the Field Health Surveillance System, both managed by the National Epidemiology Center. The former focuses on hospitalized cases, with an emphasis on those presenting as severe dengue while the latter works under the premise of being a passive system. The combination of both systems however is not able to fully capture and report all the cases and deaths of dengue, yielding an underestimate of their true value, as they rely mainly on clinical diagnosis without confirmation through laboratory diagnosis. Laboratory diagnosis utilized in the identification of dengue can be categorized into 4 general techniques: virus isolation, molecular methods, detection of viral products and serological diagnosis. The detection of the viral product non-structural protein 1 (NS1) has then become the standard for dengue diagnostics because of its high sensitivity and specificity despite its simplicity (Wang et al., 2020). The increase in vector density is observed with the creation of breeding sites for the vector due to rapid and unplanned urbanization, subsequently increasing opportunities for transmission (WHO, 2009). In the evaluation of the World Health Organization in 2010, vector control has had a significant impact on dengue prevention. Its effectiveness can be further increased when taken with appropriate community education (Yboa and Labrague, 2013). The importance of health education in the prevention of dengue is apparent as it directly affects human knowledge, attitude, and practices (KAP) (Selvarajoo et al., 2020).

In 2019, the Department of Health proposed several action plans, including case surveillance, case management, and diagnosis procedures, integrated vector management (IVM) plans, outbreak response plans, and several health promotions and advocacy projects with aims of addressing the Dengue response inefficiencies as the number of recorded dengue cases surpass the Alert and Epidemic Threshold. According to the $\mathrm{DOH}$, these action plans appeared to have been of some significant effect based on the $78 \%$ decrease in Dengue-related deaths in the first half of 2020 due to their Water-borne infectious diseases, Influenza, and Leptospirosis, including Dengue (W.I.L.D.) initiative. However, dengue cases in the Philippines remain a pervasive case of concern given how the annual Dengue case trends continue to fluctuate.

There is a need to improve the public health response taken for the prevention and control of Dengue to minimize the possibility of future epidemics to occur. This warrants the identification of gaps challenging the public health response and their causes and factors. In this, the researchers have gained an overview of the current situation and reviewed the efficiency of the response plans and advocacy projects adopted, evaluated the influence of the specific management plans, and identified the gaps in the public health that are currently implemented in the Philippines by means of a systematic review. Specific recommendations and certain factors that need further consideration and focus were also identified. 


\section{Methodology}

In order to obtain a comprehensive outlook of the public health challenges for dengue transmission and control, the researchers conducted a systematic review that utilized and reviewed data from studies, journals and literature exclusively in the Philippines or related to the Philippine setting.

\subsection{Search Strategy}

The Systematic review was conducted in accordance with the Preferred Reporting Items for Systematic Review and Meta-Analyses (PRISMA) 2020 guidelines wherein the search for literature was limited through the use of online databases. From January 31 - February 7, 2021, the researchers collated journal articles from five online databases namely Science direct, PubMed, HERDIN, Western Pacific Surveillance and Response (WPSAR), and Taylor and Francis Journal. The search string used consists of a combination of terms which are "dengue in the Philippines" and "dengue prevention and control in the Philippines."

\subsection{Screening}

The researchers conducted a screening process to determine and select the studies to be included in the review. In the initial screening stage, each database was independently screened and only journal articles published in English between January 1, 2015 and December 31, 2020 in full text were included. After initial screening, the researchers held joint discussions to accurately pinpoint and remove duplicate articles across the different online databases. The second screening stage only included journal articles with titles or abstract that focus on or are related to dengue control and prevention in the Philippines. Studies that were not focused on or related to dengue were excluded from the second screening selection.

\subsection{Eligibility}

The screened full-text papers selected from the screening process were independently assessed for eligibility to ensure that the journal articles met the inclusion criteria of the study. Journal articles with methods or results not clearly elaborated and duplicate results from different studies were excluded. In addition, meta-analysis and other systematic reviews were excluded from the review.

\subsection{Data Extraction}

After the eligibility assessment, the researchers were tasked to read the screened and collected articles and later on were each assigned a number of articles to extract data individually. The data extracted were summarized and compiled using google sheets, wherein the data was categorized into author, date publication, the primary location of the study, study duration, research theme, and identified public health challenges. The research theme categories of the articles were identified based on the excerpts from the objectives and results of the Trends in Dengue Research in the Philippines by Agrupis et al. (2019). Joint discussions were also conducted to discuss each article and its extracted data and decide whether the data extracted is correct, sufficient, and representative. 


\subsection{Risk of Bias Assessment}

In establishing transparency of the synthesized results and findings, risk of bias assessment was done by two group members by utilizing the Risk of Bias in Systematic Reviews (ROBIS) tool. This assessment tool checks in which stage of the systematic review bias was introduced and assessed the overall risk of bias in the synthesis of results and interpretation of findings. The assessment was completed in two phases (Phase 2 and Phase 3 ) in which phase 1 was not included since it was only optional and not applicable to this study.

\subsection{Synthesis of Results}

The overall synthesis of the data reviewed was descriptive as there were no studies that require statistical significance. A descriptive approach was deemed more suited for the study since the aim of the study is to provide a comprehensive review that identifies challenges, factors and recommendations on dengue control in the aspect of public health.

\section{Results and Discussion}

\subsection{General Characteristics of the Included Studies}

The search strategy initially identified 4,128 articles from different databases, of which 3,498 articles were eliminated based on their publication dates and their full-text availability. A total of 258 articles were removed, then 372 articles were screened based on titles, abstracts, language, and type of article, of which 282 were excluded, then 90 full-text articles were assessed for eligibility. After the review of the full-text studies, a total of 19 articles were selected for analysis. The details of the article selection phase results are provided in the PRISMA flowchart (Fig 1).
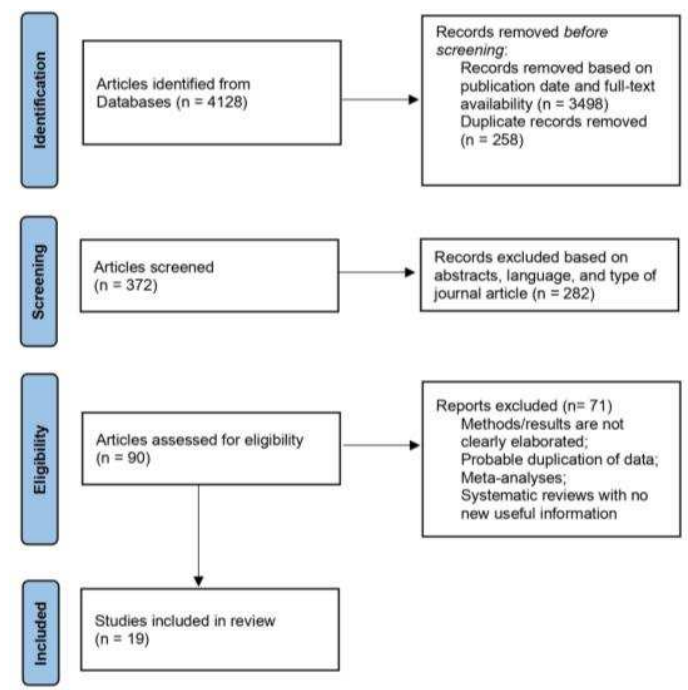

Fig. 1. Summary of the Selection Phase results 
The selected studies are then classified as follows: 5 (26.3\%) burden of disease (e.g., economic impacts of dengue); 5 (26.3\%) dengue vaccination (e.g., Dengvaxia and vaccine hesitancy); 9 (47.4\%) dengue prevention and control, which are further sub-categorized into laboratory diagnosis, community-based control programs, sociobehavioral factors, vector control and surveillance, meteorological factors and climate change. Table 1 shows the characteristics of the 19 studies that identified the different challenges in dengue transmission and control in the Philippines.

Table 1. Characteristics of the Selected Studies

\begin{tabular}{|c|c|c|c|}
\hline References & Study Area and Period & Research Theme & Identified Challenges/Key Findings \\
\hline Aumentado et al., 2015 & $\begin{array}{l}\text { Tacloban City, } \\
\text { Philippines (2014-2015) }\end{array}$ & $\begin{array}{l}\text { Dengue Prevention } \\
\text { and Control }\end{array}$ & $\begin{array}{l}\text { Dengue control relies on collaboration } \\
\text { among LGUs, DOH, and communities }\end{array}$ \\
\hline Balingit et al., 2020 & $\begin{array}{l}\text { Tarlac City, Philippines } \\
\text { (2015) }\end{array}$ & $\begin{array}{l}\text { Dengue Prevention } \\
\text { and Control - } \\
\text { Vector } \\
\text { Surveillance }\end{array}$ & $\begin{array}{l}\text { Asymptomatic infections are often } \\
\text { ignored in testing; one-step multiplex RT- } \\
\text { PCR can be used to successfully identify } \\
\text { asymptomatic strains }\end{array}$ \\
\hline Chua et al., 2019 & Philippines (July 2019) & $\begin{array}{l}\text { Dengue Prevention } \\
\text { and Control - } \\
\text { Meterological } \\
\text { Factors/Climate } \\
\text { Change }\end{array}$ & $\begin{array}{l}\text { Apparent gaps in research topics on the } \\
\text { impacts of climate change on health; } \\
\text { prioritizing health vulnerabilities and } \\
\text { health adaptation to climate change could } \\
\text { help in forming policies }\end{array}$ \\
\hline
\end{tabular}

Cheng et al., 2018

Philippines (2008-2014)

Manila, Cebu, Philippines (2008-2013)

Fitzpatrick et al., 2017

Flasche et al., 2019

Herbuela et al., 2019

Iguchi et al., 2018

Larson et al., 2018

Lopez et al., 2020
Philippines, Brazil, Disease Burden Columbia, Malaysia, Mexico, Thailand (2017)

Philippines (2016-2019) Dengue Vaccine

Metro Manila, Philippines (JulyNovember 2017)

Davao region, Philippines (2011-2015)

Philippines (2015-2018)

Cebu,Philippines (May-
Dengue Prevention and Control Sociobehavioral factors

Dengue Prevention and Control Vector Surveillance

Dengue Vaccine

Dengue Prevention and Control -
Direct medical costs of dengue incur significant socioeconomic costs; dengue's economic burden in the Philippines is expected to remain high

Costs due to underreporting of dengue cases necessitates the need for evaluation of dengue surveillance systems

There is a challenge of maintaining vector control in the absence of an effective vaccine program and outbreak responses

Dengvaxia's potential is not to be discounted solely due to the failure in screening procedures of seronegative individuals

Knowledge and attitude of patients did not correlate with practices against dengue

Changes exhibited by meteorological factors affect people's health by increasing number of vector-borne diseases

The dengue vaccination scare affected public confidence in the vaccine and other clinic-based treatments.

Pre-vaccination screening methods are 


\begin{tabular}{|c|c|c|c|}
\hline & June, 2017) & $\begin{array}{l}\text { Laboratory } \\
\text { Diagnosis }\end{array}$ & inaccessible and impractical \\
\hline Mehta et al., 2019 & $\begin{array}{l}\text { Cebu, Philippines } \\
\text { (2019) }\end{array}$ & $\begin{array}{l}\text { Dengue Prevention } \\
\text { and Control - } \\
\text { Laboratory } \\
\text { Diagnosis }\end{array}$ & $\begin{array}{l}\text { Available methods for diagnosis of DENV } \\
\text { are either not suitable in communities that } \\
\text { lack resources, or have low sensitivity and } \\
\text { specificity }\end{array}$ \\
\hline Migriño et al., 2020 & $\begin{array}{l}\text { San Miguel, Manila, } \\
\text { Philippines (2020) }\end{array}$ & Dengue Vaccine & $\begin{array}{l}\text { Main reason for vaccine hesitancy is } \\
\text { negative media information and concerns } \\
\text { on safety and side effects }\end{array}$ \\
\hline Nealon et al., 2016 & $\begin{array}{l}\text { Indonesia, Malaysia, } \\
\text { Philippines, Vietnam } \\
\text { (June-December 2011) }\end{array}$ & Disease Burden & $\begin{array}{l}\text { The national surveillance system fails to } \\
\text { estimate the burden of the disease }\end{array}$ \\
\hline Pineda-Cortel et al., 2019 & $\begin{array}{l}\text { NCR, Region II, Region } \\
\text { 1, Calabarzon, } \\
\text { Philippines (2018-2019) }\end{array}$ & $\begin{array}{l}\text { Dengue Prevention } \\
\text { and Control - } \\
\text { Meteorological } \\
\text { Factors/Climate } \\
\text { Change }\end{array}$ & $\begin{array}{l}\text { The association between dengue incidence } \\
\text { and climatic variables can be used to } \\
\text { create an early warning model for } \\
\text { effective prevention and control strategies }\end{array}$ \\
\hline Reyes et al., 2020 & $\begin{array}{l}\text { Philippines (January- } \\
\text { March 2017) }\end{array}$ & Dengue Vaccine & $\begin{array}{l}\text { Perceptions of primary caregivers on } \\
\text { dengue vaccination imply their overall } \\
\text { vaccine willingness and reflect their trust } \\
\text { to the health care system }\end{array}$ \\
\hline Salazar et al., 2017 & $\begin{array}{l}\text { Muntinlupa City, } \\
\text { Philippines (2007) }\end{array}$ & $\begin{array}{l}\text { Dengue Prevention } \\
\text { and Control - } \\
\text { Sociobehavioral } \\
\text { factors }\end{array}$ & $\begin{array}{l}\text { Differences in attitude among individuals } \\
\text { in a community could affect the execution } \\
\text { of control programs }\end{array}$ \\
\hline Solon \& Panelo, 2018 & Philippines (2018) & Burden of Disease & $\begin{array}{l}\text { Costing was difficult due to lack of data } \\
\text { on serologic prevalence in the Philippines }\end{array}$ \\
\hline Valido et al., 2018 & $\begin{array}{l}\text { Quezon City, } \\
\text { Philippines (November } \\
\text { 2017-September 2018) }\end{array}$ & Dengue Vaccine & $\begin{array}{l}\text { The negative vaccine media coverages } \\
\text { induced fear for the vaccine, regret, and } \\
\text { guilt in participation and distrust on the } \\
\text { vaccination program }\end{array}$ \\
\hline
\end{tabular}

\subsection{Current Dengue Prevention and Control Programs}

Most of the dengue awareness and transmission reduction programs of the NDCP-DOH have been primarily focused on eradicating or reducing natural and artificial vector breeding habitats through community mobilization and involvement in dengue awareness activities. This involves the implementation of programs by local government units and collaboration amongst national authorities to prevent and control dengue epidemics. For instance, the 4s strategy was implemented along with fogging, canal treatment, mosquito larvae traps, and health awareness programs.

\subsection{Factors Affecting Dengue Transmission and Control}

Eight significant factors that considerably impact the public health response to dengue management, control, and treatment based on the studies reviewed were identified and are as follows: 


\subsubsection{Disease Burden}

The growing dengue cases in the Philippines may be attributed to environmental risk factors, insufficient public health infrastructure, inconsistent preventative measures, and a lack of effective monitoring systems. Along with the rise in dengue incidence in the Philippines comes an increase in medical costs, estimated to be between PhP 5.4 billion and PhP 15 billion yearly (Cheng et al., 2018). This only suggests that dengue fever may have a significant economic impact on the country through increased health care expenses and consequent loss of productivity due to disease and mortality, as well as potential reductions in tourism (Edillo et al., 2015). In addition, gaps in the surveillance of the disease in the country have resulted in a projected underreporting rate of $86.72 \%$ and an adjustment factor of 7.2 , mainly due to the reliance of surveillance systems in the Philippines on disease reporting units from barangay health centers and rural health centers, with the current system used focusing on hospitalized cases and case classification (dengue with or without major complications). Underreporting, however, is seen due to how passive surveillance is based mainly on clinical diagnosis without the utilization of laboratory confirmations. Consolidating the underreporting rate and adjustment factor in the disease surveillance, the annual reported case in 2012 of 117,065 was adjusted to 842,867 cases resulting in a total aggregate cost of $\$ 345$ million. The economic burden of dengue is then estimated to represent $0.15 \%$ of the total GDP of the Philippines in 2012. These accumulating costs for the provision of medical care and dengue, in general, due to the underreporting of cases highlight the need to evaluate further current dengue surveillance systems and the dengue control strategies in the Philippines (Edillo et al., 2015). Furthermore, the use of several models and macro costing methodologies such as those used in vaccination programs and trials (Nealon et al., 2016), basic surveillance models (Solon \& Panelo, 2018), and the Markov model (Fitzpatrick et al., 2017) may be used to establish the economic and disease burden of dengue. For instance, dengue surveillance in both vectors and humans and its relationship with vector control were established by the Markov model while estimating the economic burden of dengue. Nevertheless, if inconsistent case definitions and surveillance systems are continued to be used, the generation of consistent burden estimates and increased health costs will remain a challenge (Solon \& Panelo, 2018).

\subsubsection{Dengue Prevention and Control}

There are a range of factors influencing the transmission, control, and prevention activities on dengue in the Philippines. These factors generally include meteorological factors, ecological factors, biological factors, social factors, and the knowledge, attitude, and practice of a population about dengue (Salazar et al., 2017).

Dengue infection outbreaks are common following natural-occurring disasters such as typhoons with subsequent floods since it provides the potential for a large increase of mosquito breeding sites. For instance, when Typhoon Haiyan hit the Philippines in 2013, the damages of several infrastructures together with consistent rainfall that lasted for weeks led to excessive accumulation of stagnant water, which then led to a significant increase in mosquito population bringing a higher risk of dengue transmission. Responses such as rapid establishment of an effective surveillance system and targeted vector control were able to reduce the transmission of dengue. Moreover, the use of rapid diagnostic test kits for early diagnosis allowed the determination of the proportion of dengue cases that were RDT-positive. The daily reports of dengue also helped in the early detection of cases, thereby increasing the targeted vector control efforts. Collaboration among the local government, national government, and communities are necessary to ensure that targeted responses are rapidly established (Aumentado et al., 2015). 


\subsubsection{Community-based Control Programs}

Budget constraints and a lack of dedicated personnel contribute to the lack of adequate and sustainable dengue prevention and control initiatives. For instance, certain dengue-related activities at the barangay and school levels have also been discontinued, and information dissemination campaigns and activities about dengue prevention and control have been conducted on an occasional basis. Among lectures on various diseases conducted in health centers, dengue was classified as a sub-topic that is only emphasized during the rainy season. Furthermore, the lack of programs and coordination between the local community and public officials often results in unfulfilled routine campaigns for community cleanliness in different barangays and an unorganized system for storing water supply in individual households (Salazar et al., 2017). Thus, campaigns regarding dengue awareness that involve members of the community are recommended. Suggested programs that can be implemented by the LGUs are the following: spraying of insecticides, health education regarding dengue, and practice on the proper disposal of unused or used containers, and proper cleaning and monitoring of all water storage containers in the community (Salazar et al., 2017). Routine inspection of healthcare workers to the communities at least once a month is also recommended. Lastly, the national government should continue to address social, and community factors and transmission-enabling behaviors as the National Dengue Control and Prevention Program's primary mandate requires (Salazar et al., 2017). The success of the community-based control programs and strategies to be implemented by the LGUs relies on the knowledge, attitude, and practice of the community members, which emphasizes the need for information dissemination and creation of additional programs that will allow individuals to identify and implement dengue preventive measures on their own. This, in turn, would also bring awareness to the whole population (Salazar et al., 2017).

\subsubsection{Sociobehavioral Factors}

Dengue fever epidemiology is strongly associated with individuals' daily habits and activities, making it vital to analyze persons' knowledge, attitudes, and practices (KAP) (Herbuela et al., 2019). Studies show that having a comprehensive knowledge of dengue may not necessarily result in a behavioral change toward dengue prevention strategies. For instance, there is a significant correlation discovered between knowledge and attitude domains among hospitalized patients as a result of their knowledge about dengue symptoms, transmission, and prevention practices acquired during hospitalization and possible encounters with healthcare providers, which in return have influenced their beliefs about dengue. However, neither knowledge nor attitude was correlated to dengue prevention practices (Herbuela et al., 2019). At the community level, disparities in KAP scores are influenced by information provided by media sources (e.g., television, radio, and others), lectures delivered by local health centers, training for preventive activities provided by LGUs, and community members' attitudes toward the health workers who facilitated the training and the government's health-related efforts (Salazar et al., 2017). Additionally, it was observed that certain activities were carried out on an occasional basis, resulting in a considerable decrease in KAP scores. These disparities among community members' attitudes affect the risk of dengue transmission and the application of community-based control methods (Salazar et al., 2017). Furthermore, respondents have developed a reliance on government programs for their dengue prevention safety, resulting from their belief that the government is primarily responsible for dengue prevention (Salazar et al., 2017). These findings highlight the very importance of behavioral change in ensuring that individuals' knowledge and attitudes regarding dengue substantially impact their prevention behaviors. Moreover, existing programs focus heavily on increasing public awareness and understanding of dengue fever above physical activities, when health programs should prioritize behavioral change through transforming information and attitudes into effective behaviors (Herbuela et al., 2019) to 
effectively implement disease prevention and control in the community, which could be achieved through comprehensive programs such as the Communication for Behavioral Impact (COMBI) and Health Belief Model (HBM).

\subsubsection{Vector Control Strategies}

In the study of Salazar et al. (2017), community members in Muntinlupa City classified fogging as the most evident government intervention being implemented in reducing the number of dengue vectors, followed by checking of water containers, treatment of canals with larvicides, information dissemination campaigns, and trapping of mosquito larvaes. However, fogging in communities has been overshadowed by politicians who implement political fogging campaigns during election, which caused the majority of the residents to have misconceptions about the use of fogging in dengue control (Salazar et al., 2017). Moreover, the residents continuously relied on the outdoor space spraying by their LGUs, making them dependent on government interventions. Therefore, this implies that more persuasive health-related campaigns may be needed, particularly in the importance of good practices for control of mosquito population, and in correcting misconceptions about dengue. Furthermore, the control of dengue transmission heavily depends on the extermination of the vector population which is mainly through conducting biological vector control plans. Comprehensive vector control activities such as fogging, larviciding, and search-and-destroy activities implemented on areas that were identified to be mosquito breeding sites and other debris and garbage collection areas has lessened the densities of mosquitoes and flies in the affected areas of Typhoon Haiyan, and are therefore recommended (Aumentado et al., 2015). Spraying has also been found effective in decreasing mosquito populations during the aforementioned typhoon. However, it is not recommended on a large scale except during outbreaks (Aumentado et al., 2015).

\subsubsection{Vector Surveillance}

An effective control of dengue vectors must rely on accurate detection of mosquito-borne infections, along with adequate knowledge on the vector population and densities (Muller et al., 2017). The recent advancements in nucleic acid amplification tests (RT-PCR) enabled the direct detection of DENV RNA in field-collected mosquitoes, despite the low infection rate of dengue virus in adult female Aedes aegypti mosquitoes. However, no current consensus on the optimal pool size for mosquito screening has been established, which may result in erroneous infection rate estimations. Monitoring dengue virus infection rate through employing an individual-vector based approach was proven to be functional due to its precision and accuracy in the estimation of the dengue infection rate. Through the individual-based DENV detection utilizing the RT-PCR assay, direct DENV RNA sequencing from individual mosquitoes is made possible. This can then potentially prove useful for vector-based DENV surveillance through its identification of DENV genotypes and serotypes in both patients and vectors/mosquitos. Furthermore, the field-collected mosquitos' viral data can then be incorporated into patient-based dengue surveillance programs thus helping increase the sensitivity by improving the predictions and prevention measures for outbreaks of dengue through the detection of the silently circulating DENV (Balingit et al., 2020).

\subsubsection{Laboratory Diagnosis}

Early identification and diagnosis of dengue are essential to identify cases that can lead to death and help public health agencies implement appropriate prevention and control measures. However, the currently 
available diagnostic techniques are either impractical (e.g., virus isolation) and inaccessible (Mehta et al., 2019), particularly in endemic countries or areas lacking adequate resources for expensive instruments, trained personnel, suitably equipped laboratories (e.g., PCR-based tests), or limited by sensitivity, specificity, and cross-reactivity (e.g., RDTs that detect NS1, IgG, or IgM). With all these constraints, Mehta et al. (2019) validated a field-ready diagnostic test for dengue virus detection directly from blood or plasma utilizing a portable Open qPCR Thermocycler, without the need for performing RNA extraction. This technique was developed for use in hyperendemic areas that lack the resources necessary to conduct the recommended laboratory diagnosis for DENV (Mehta et al., 2019), while overcoming the issue of cross-reactivity with other viruses. Utilizing an inexpensive, specific, and easy method could be used for clinical management and dengue surveillance, which in return would provide early intervention to control epidemics.

Dengue serostatus is also crucial for pre-vaccination screening; however, current methods (e.g., Neutralization tests) are expensive, time-consuming, and require advanced technical skills and equipment (Lopez et al., 2020). Therefore, the development and validation of other laboratory techniques that can be used to establish serostatus for pre-vaccination that would surpass the mentioned shortcomings is recommended.

\subsubsection{Meteorological Factors and Climate Change}

Meteorological factors namely temperature, rainfall and humidity are considered to be one of the causes for the increase of dengue incidence worldwide. Changes exhibited by these factors influence the health status of an individual through the spread of vector-borne diseases (Iguchi et al., 2018). For instance, temperature affects the adult mosquitoes' behavior and maturation (Iguchi et al., 2018) by shortening the developmental cycle of the mosquitoes, thus, also shortening the incubation period of the virus (Pineda-Cortel et al., 2019). A rise in temperature would allow a rapid rate of reproduction, which would subsequently result in an increased capacity of transmission potential of dengue fever. In addition, rainfall provides a conducive site for the propagation of mosquitoes, wherein, dengue infection cases in moderate rainfall are higher compared to heavy rainfall in which the eggs, larvae, and pupae are flushed away affecting the abundance of mosquitoes. Vectors are also able to reproduce in the dry season due to the presence of water storages that are favorable for mosquito growth and development. High humidity is also a contributing factor that increases the longevity of adult mosquitoes as well as the shortening of the viral incubation period. (Iguchi et al., 2018). Considering all these factors, there is a lack of research topics that focus on the impact of climate change on health. Thus, prioritizing health vulnerabilities and health adaptation to climate change countries considered vulnerable to extreme effects of climate change is recommended as it could help in the formation of mitigation policies to enhance dengue prediction for better vector control and management of dengue in the future (Chua et al., 2019).

\subsubsection{Dengue Vaccination}

With the continuous prevalence of Dengue cases in the Philippines along with its high burden both economically and in terms of public health, it is only logical to expect the Philippine government to introduce Dengue vaccination programs to the public. This was done in 2016 with the Dengvaxia vaccine that would have supposedly given immunity to all 4 serotypes of Dengue. The Dengvaxia program however, became a downfall rather than being the intended advocacy filled program with an ultimatum of reducing dengue prevalence had it gone according to plan, as a decline in official public health trust and an increase in vaccine hesitancy was observed (Larson et al., 2018). 


\subsubsection{Vaccine Hesitancy}

Dengue vaccine hesitancy was heavily exacerbated due to the Dengvaxia serostatus implication incident in the Philippines. Several factors were involved in this said exacerbation which included attitudes towards vaccination of primary caregivers, knowledge on dengue fever, previous exposure to dengue, and negative media exposure during the time of the controversy among others. Reyes et al. (2020), Valido et al. (2018) and Migriño et al. (2020) all recommend adequate trust building and communication between healthcare providers. Thus, it can be implied that better government-community communication strategies and improved pre-vaccination serostatus screening should be practiced and implemented. Furthermore, Valido et al. (2018) strongly argue that communication strategies that would efficiently inform parents or primary caregivers of the benefits and safety of the vaccine, and that would gain the trust and confidence of the society would be vital in reducing dengue vaccine hesitancy.

\subsubsection{Potential of Dengue Vaccine}

The emphasis on the potential role of Dengvaxia in public health in the absence of other alternatives and if properly administered and overseen by health officials is accompanied by challenges on communication with the public for the healthcare providers, with the recommendation of strict following of a pre-vaccination screening strategy. This strategy however, would also pose more economical challenges including increased cost in logistics, communication, and the lack of sensitive and specific POCT capable of assessing serostatus of potential vaccinees (Flasche et al., 2019).

\subsection{Discussion}

The challenges that Dengue infection and transmission pose towards the Philippine public health system are deemed more complex than simply its viral pathogenesis and mechanisms of infection. In this systematic review, the researchers were able to collect and summarize updated information on the factors concerning the challenges to Dengue prevention and control here in the Philippines. These factors include disease burden, disease prevention and control, vector control and surveillance, Dengue vaccination, sociobehavioral factors, laboratory diagnosis, community-based control programs, and climatic and environmental factors. These factors highlight the importance of looking into aspects other than the viral pathogenesis of dengue in order to effectively mitigate and control the spread of the disease.

Starting with Disease burden whose main challenge was the underreporting and underestimation of dengue cases that increases overall healthcare costs. The annual cost of dengue (i.e. allocation for diagnostic techniques and methods, direct medical costs) presented in the different models due to underreporting of cases and of dengue in general only highlight the need to further evaluate the current dengue surveillance systems, as well as the dengue control strategies in the Philippines. It is important to note that with improved dengue surveillance and dengue control strategies, estimates of the incidence of dengue episodes and the cost of dengue treatment per case will be refined. In order to improve this estimation, the use of immediate, shortterm and long-term modifications are recommended (Shepard et al., 2014). These included the documentation of ambulatory visits before and after hospitalization, organization of data, improvement of RDTs and more frequent collection and analysis of seroprevalence data. The use of covariates in the documentation of dengue cases (Undarraga, et al., 2013) along with comparing cohort study data with national surveillance data (Wichmann et al., 2011) were other methods seen to be effective in estimating dengue burden. 
Dengue prevention and control is a factor that concerns how dengue surveillance systems and targeted vector control measures must be established and strengthened given how infection outbreaks are common following natural disasters such as typhoons. Furthermore, linkages among government agencies and communities are also beneficial and effective for countering challenges with successful control programs $(\mathrm{Ng}$, 2011), similar to the prompt collaboration between the LGUs, DOH, and the communities involved after Typhoon Haiyan, which was also applicable in the community-based control programs. Following that, routine or regular visits and inspections from assigned healthcare workers to individual households are then recommended to help induce an active and consistent participation among individuals in a more personal manner (Selvarajoo et al., 2020) along with the implementation of programs that would develop critical thinking and preventive practice for people for community-based control programs. This is since Dengue requires community participation to effectively combat the factors that propagate its spread, which could be done if sufficient budget is provided (Carandang et al., 2017), similar to how the DOH was able to implement an enhanced $4 \mathrm{~s}$ strategy and disseminate different materials for destroying vector breeding sites and selfprotection earlier last year.

The sociobehavioral factor challenge mainly revolves around how having the knowledge about dengue prevention doesn't always translate into good practice which could even increase the risk due to carelessness and false complacency, and on the dependence of people towards government interventions alone for their own safety. These therefore emphasize the need for health education programs that strengthen IEC campaigns on Dengue prevention (Yboa and Labrague, 2013) and at the same time can invoke behavioral change aside from merely informing the public (Selvarajoo, 2020) wherein invoking actual behavioral change is the key difference between the effectivity of these programs or campaigns directed towards communities. Meanwhile, individual-vector based surveillance and emphasis on eradication of vectors rather than only identification are highlighted for improved vector surveillance (Rather et al., 2017), whereas persuasive campaigns on vector control and further research on control methods are both recommended actions for vector control. Larviciding along with the $4 \mathrm{~s}$ strategy involving fogging have also shown their effectiveness, but it must be managed accordingly while avoiding indiscriminate fogging since insecticide fogging causes high adverse effects on insects other than mosquitos in the environment (Abeyasuriya et al., 2017). Thus, proper use and management of these methods are emphasized and must be observed.

Further research and development of a cost-effective, efficient, and less complex diagnostic method is the main recommendation for laboratory diagnosis similar to how the DOH claimed the NAAT-LAMP is as implemented earlier last year. This is due to how current sensitive and specific tests available such as virus isolation and nucleic acid detection methods pose the challenges of being laborious, time consuming, and costly, which limits their routine use in dengue endemic countries as they are not always an option in remote areas where dengue is endemic (Muller et al., 2017). On the other hand, the more user-friendly, easily accessible, and low-cost Rapid Diagnostic Tests/kits then pose the challenge of lacking sensitivity and specificity particularly in the diagnosis of secondary infections and its cross-reactivity with other flaviviruses. This along with better serostatus diagnosis for pre-vaccination screening are both recommended (Luo et al., 2019).

Meanwhile, inevitable climate and meteorological variables that were optimal conditions for vector growth and shortened viral incubation periods including having high humid temperature in the area with seasonal and frequent moderate rainfall to go along with crowded urban areas that promote vector proliferation all being present here in urban areas of the Philippines further emphasizes the importance of vector surveillance and control, wherein climatic data can prove vital in the early prediction and mitigation of 
vector-borne diseases in the Philippines (Lee et al., 2018). Further research on climate change impacts on health are also crucial and recommended to continuously lessen the burden of vector-borne diseases amidst the additional challenges posed by climate change and changes in environmental or social determinants (Campbell-Lendrum et al., 2015).

Lastly, vaccine hesitancy and lessened public trust towards health officials brought by the Dengvaxia controversy can be alleviated through improved communication strategies involving vaccine makers, healthcare officials, and the public concerning the vaccine's effectiveness and explanation of the past controversy. Advocating towards the seropositive individuals could also prove to be more beneficial instead of politicizing issues, this is similar to what Brazil did with the serostatus finding to avoid public panic (Laroza, 2019), but the subject remains more complex seeing as to how children's lives were claimed to be lost and at danger due to Sanofi's late serostatus implication. Better and more accessible means serostatus diagnosis for pre-vaccination screening in future vaccine programs are also needed given the vaccination program's potential if done with a strictly implemented pre-vaccination screening phase.

Identifying and addressing the critical gaps in dengue prevention and control would aid in the accurate assessment of the burden of the disease and implement a better dengue disease response. Describing the current challenges the country is facing regarding dengue prevention and control can then provide a template for the development of research that would aim to address such challenges. Identifying the different factors elaborated in this study that hinder the continuous efforts to prevent and control the spread of dengue would help determine where current programs are lacking, or where more recent programs that were not included in this review could further improve upon and could help direct their focus into areas which need more emphasis. Thus, the findings from this study could provide the policy-makers with significant information that would aid the rational decision-making process regarding dengue preventive and control efforts.

This systematic review was able to identify the major factors that contribute to the challenges on Public Health control and prevention of Dengue and identify certain areas with gaps along with the different corresponding recommendations. Despite being able to identify a number of challenges and recommendations, this review is in no way fully comprehensive and complete. The researchers solely utilized the use of electronic databases that had filter features in their search systems and collected studies that were free to access, whereas there are bound to be more studies and articles in different sources such as in physical libraries and in other electronic databases. This also inhibited the systematic review's nature of covering unpublished works or "grey literature" as well since we only relied on electronic databases which only consisted of published works while still excluding editorials, news articles, and such. There is also no registered protocol for this review, which could be developed by future studies who wish to conduct a similar review. The likelihood of having more published literature concerning more recent public health activities and programs beyond this review's timeframe also limited its comprehensiveness and further recommends future research regarding the same topic with updated contexts. We also limited our search to individual and original studies and excluded other systematic reviews and meta-analyses given this study's qualitative nature as opposed to being a meta-analysis.

\section{Conclusion}

Understanding the prevention and control of Dengue in the Philippines involves an elaborate public health investigation and review of several different factors not exclusively limited to its viral pathogenesis and 
mechanisms of infection. For a more productive and efficient means of lessening the prevalence of Dengue, several aspects originating from individual behavior and practices of people up until coordination of LGUs and communities and budget allocation of national-level programs must all be properly aligned, and to achieve this, proper focus on the right aspects and areas needing improvement must be observed.

For future studies, expanding the search criteria or databases when collecting articles including editorials, news articles, journal logs of researchers, and other works otherwise known as "grey literature" is recommended for a more comprehensive review. Future research may also consider registering a protocol similar to the criteria and objectives adhered throughout this review. Conducting further research with similar objectives in the future is also recommended to include more recently published journals and studies that were not yet published during this review's timeframe or did not have published literature in the databases used during the conducting of this review. It is also recommended that further investigation and exploring of the individual factors mentioned be done as it could aid in providing more specific recommendations through perhaps their identification of several more specific current programs and interventions that may have gaps similar to but more specific than what has been identified in this study.

\section{Acknowledgements}

The proponents of this study would like to express their deep and sincere gratitude to their adviser, Asst. Prof. Gamaliel Issamar De Vera, RMT, MSMT for imparting his knowledge and invaluable guidance to the proponents throughout the research, their parents and families, who supported them morally and financially, and finally, to the Lord Almighty, for His unending Grace and Wisdom that pushed us to the completion of this research.

\section{References}

Abeyasuriya, K., Nugapola, N., Perera, M., Karunaratne, W., \& Karunaratne, S. (2016). Effect of dengue mosquito control insecticide thermal fogging on non-target insects. International Journal Of Tropical Insect Science, 37(01), 11-18. doi: $10.1017 / \mathrm{s} 1742758416000254$

Agrupis KA, Ylade M, Aldaba J, Lopez AL, Deen J (2019) Trends in dengue research in the Philippines: A systematic review. PLoS Negl Trop Dis 13(4): e0007280. https://doi.org/10.1371/journal.pntd.0007280

Aumentado, C., Cerro, B. R., Olobia, L., Suy, L. L., Reyes, A., Kusumawathie, P. H., Sagrado, M., Hall, J. L., Abeyasinghe, R., Foxwell, A. R., \& Vestergaard, L. S. (2015). The prevention and control of dengue after Typhoon Haiyan. Western Pacific surveillance and response journal : WPSAR, 6 Suppl 1(Suppl 1), 60-65. https://doi.org/10.5365/WPSAR.2015.6.3.HYN_018

Balingit, J. C., Carvajal, T. M., Saito-Obata, M., Gamboa, M., Nicolasora, A. D., Sy, A. K., Oshitani, H., \& Watanabe, K. (2020). Surveillance of dengue virus in individual Aedes aegypti mosquitoes collected concurrently with suspected human cases in Tarlac City, Philippines. Parasites \& vectors, 13(1), 594. https://doi.org/10.1186/s13071-020-04470-y

Bravo, L., Roque, V. G., Brett, J., Dizon, R., \& L'azou, M. (2014). Epidemiology of Dengue Disease in the Philippines (2000-2011): A Systematic Literature Review. PLoS Neglected Tropical Diseases, 8(11). doi: 10.1371/journal.pntd.0003027

Campbell-Lendrum, D., Manga, L., Bagayoko, M., \& Sommerfeld, J. (2015). Climate change and vector-borne diseases: what are the implications for public health research and policy? Philosophical transactions of the Royal Society of London. Series B, Biological sciences, 370(1665), 20130552. https://doi.org/10.1098/rstb.2013.0552sheparlar

Carandang, R., Valones, A., Valderama, M., Cotoco, K., \& Asis, E. (2017). Community-based approach for dengue prevention and control in Sta. Cruz, Laguna, Philippines. International Journal Of Community Medicine And Public Health

Cheng, K. G.,Lam, H. Y., Rivera, A. S., Tumanan-Mendoza, B. A., Alejandria, M. M., \& Wu, D. B(2018).Estimating the burden of dengue in the Philippines using a dynamic transmission model. Acta Medica Philippina, 52(2), 153-159

Chua, P. L., Dorotan, M. M., Sigua, J. A., Estanislao, R. D., Hashizume, M., \& Salazar, M. A. (2019). Scoping Review of Climate Change and Health Research in the Philippines: A Complementary Tool in Research Agenda-Setting. International journal of environmental research and public health, 16(14), 2624. https://doi.org/10.3390/ijerph1614

Edillo, F. E., Halasa, Y. A., Largo, F. M., Erasmo, J., Amoin, N. B., Alera, M., Yoon, I. K., Alcantara, A. C., \& Shepard, D. S. (2015). Economic cost and burden of dengue in the Philippines. The American journal of tropical medicine and hygiene, 92(2), 360-366. https://doi.org/10.4269/ajtmh.14- 0139 
Fitzpatrick, C., Haines, A., Bangert, M., Farlow, A., Hemingway, J., \& Velayudhan, R. (2017). An economic evaluation of vector control in the age of a dengue vaccine. PLoS neglected tropical diseases, 11(8), e0005785. https://doi.org/10.1371/journal.pntd.0005785

Flasche, S., Wilder-Smith, A., Hombach, J., \& Smith, P. G. (2019). Estimating the proportion of vaccine-induced hospitalized dengue cases among Dengvaxia vaccinees in the Philippines. Wellcome open research, 4 , 165. https://doi.org/10.12688/wellcomeopenres.15507.1

Herbuela, V. R. D. M., de Guzman, F. S., Sobrepeña, G. D., Claudio, A. B. F., Tomas, A. C. V., Reyes, C. M. A. D., ... Watanabe, K. (2019). Knowledge, attitude, and practices regarding dengue fever among pediatric and adult in-patients in metro Manila, Philippines. International Journal of Environmental Research and Public Health, 16(23). https://doi.org/10.3390/ijerph16234705

Iguchi, J. A., Seposo, X. T., \& Honda, Y. (2018). Meteorological factors affecting dengue incidence in Davao, Philippines. BMC public health, 18(1), 629. https://doi.org/10.1186/s12889-018-5532-4

Laroza, C. (2019, February 12). What Brazil Did Right About Dengvaxia (That the Philippines Didn't). Flip Science. https://www.flipscience.ph/health/dengvaxia-scare-philippines-ii/.

Larson, H. J., Hartigan-Go, K., \& de Figueiredo, A. (2019). Vaccine confidence plummets in the Philippines following dengue vaccine scare: why it matters to pandemic preparedness. Human vaccines \& immunotherapeutics, 15(3), 625-627. https://doi.org/10.1080/21645515.2018.1522468

Lee, H. \& Kim, J. \& Lee, S. \& Lee, C. (2018 June 18). Potential effects of climate change on dengue transmission dynamics in Korea. PLoS One, 13(6). doi: 10.1371/journal.pone.0199205

Lopez, A. L., Adams, C., Ylade, M., Jadi, R., Daag, J. V., Molloy, C. T., Agrupis, K. A., Kim, D. R., Silva, M. W., Yoon, I. K., White, L., Deen, J., \& de Silva, A. M. (2021). Determining dengue virus serostatus by indirect IgG ELISA compared with focus reduction neutralisation test in children in Cebu, Philippines: a prospective population-based study. The Lancet. Global health, 9(1), e44-e51. https://doi.org/10.1016/S2214-109X(20)30392-2

Luo et al. (2019, June). Rapid diagnostic tests for determining dengue serostatus: a systematic review and key informant interviews. Clinical Microbiology and Infection, 25(6), 659-666. https://doi.org/10.1016/j.cmi.2019.01.002

Mehta, N., Perrais, B., Martin, K., Kumar, A., Hobman, T. C., Cabalfin-Chua, M. N., Donaldo, M. E., Siose Painaga, M. S., Gaite, J. Y., Tran, V., Kain, K. C., Hawkes, M. T., \& Yanow, S. K. (2019). A Direct from Blood/Plasma Reverse Transcription-Polymerase Chain Reaction for Dengue Virus Detection in Point-of-Care Settings. The American journal of tropical medicine and hygiene, 100(6), 1534-1540. https://doi.org/10.4269/ajtmh.19-0138

Migriño, J., Jr., Gayados, B., Birol, K., De Jesus, L., Lopez, C., Tolosa, W., . . Tulagan, G. (2020). Factors Affecting Vaccine Hesitancy among Families with Children 2 Years Old and Younger in Two Urban Communities in Manila, Philippines. Western Pacific Surveillance and Response, 11. doi:doi: 10.5365/wpsar.2019.10.2.006.

Muller, D. \& Depelsenaire, A. \& Young, P. (2017, March 1). Clinical and Laboratory Diagnosis of Dengue Virus Infection. The Journal of Infectious Diseases, 215(2), 89-95. https://doi.org/10.1093/infdis/jiw649

$\mathrm{Ng}$, L. (2011). Challenges in dengue surveillance and control. Western Pacific Surveillance And Response, 2(2), e1-e1. doi: 10.5365/wpsar.2011.2.2.001ther

Nealon, J., Taurel, A. F., Capeding, M. R., Tran, N. H., Hadinegoro, S. R., Chotpitayasunondh, T., Chong, C. K., Wartel, T. A., Beucher, S., Frago, C., Moureau, A., Simmerman, M., Laot, T., L'Azou, M., \& Bouckenooghe, A. (2016). Symptomatic Dengue Disease in Five Southeast Asian Countries: Epidemiological Evidence from a Dengue Vaccine Trial. PLoS neglected tropical diseases, 10(8), e0004918. https://doi.org/10.1371/journal.pntd.0004918

Pineda-Cortel, M. \& Clemente, B. \& Nga, P. (2019 February 1). Modeling and predicting dengue fever cases in key regions of the Philippines using remote sensing data. Asian Pacific Journal of Tropical Medicine, 12 (2), 60-66. DOI: 10.4103/1995-7645.250838

Polwiang S. (2020). The time series seasonal patterns of dengue fever and associated weather variables in Bangkok (2003-2017). BMC infectious diseases, 20(1), 208. https://doi.org/10.1186/s12879-020-4902-6

Rather, I. A., Parray, H. A., Lone, J. B., Paek, W. K., Lim, J., Bajpai, V. K., \& Park, Y. (2017). Prevention and Control Strategies to Counter Dengue Virus Infection. Frontiers in Cellular and Infection Microbiology, 7. doi:10.3389/fcimb.2017.00336

Reyes, S. L., Lee, K. G., Pedron, M. L., Pimentel, J. T., \& Pinlac, P. V. (2020). Factors associated with the willingness of primary caregivers to avail of a dengue vaccine for their 9 to 14-year-olds in an urban community in the Philippines. Vaccine, 38(1), 54-62. doi: https://doi.org/10.1016/j.vaccine.2019.10.001

Salazar, F. V., Cruz, E. I., Bugayong, P. J., Fulache-Aligato, M. , Marco, J., Merndoza, Y. , (2017).Determinants of transmission risk and the role of vector pupal presence in the development of integrated approaches to dengue control in Muntinlupa City, The Philippines. Southeast Asian Journal of Tropical Medicine and Public Health, 48(5), 955-974

Selvarajoo et al. (2020 June). Knowledge, attitude and practice on dengue prevention and dengue seroprevalence in a dengue hotspot in Malaysia: A cross-sectional study. 10.1038/s41598-020-66212-5

Solon, O., \& Panelo, C. (2018). PRM10 An Approach to Costing Medical Care Needs Under Uncertainty: The Case of Dengue Mass Vaccination in the Philippines. Value in Health, 21. doi: https://doi.org/10.1016/j.jval.2018.07.724

Shepard, D. S., Undurraga, E. A., Betancourt-Cravioto, M., Guzmán, M. G., Halstead, S. B., Harris, E., Mudin, R. N., Murray, K. O., Tapia-Conyer, R., \& Gubler, D. J. (2014). Approaches to refining estimates of global burden and economics of dengue. PLoS neglected tropical diseases, 8(11), e3306. https://doi.org/10.1371/journal.pntd.0003306

Undurraga, E. A., Halasa, Y. A., \& Shepard, D. S. (2013). Use of expansion factors to estimate the burden of dengue in Southeast Asia: a systematic analysis. PLoS neglected tropical diseases, 7(2), e2056. https://doi.org/10.1371/journal.pntd.0002056 
Valido, E. M., Laksanawati, I. S., \& Utarini, A. (2018). Acceptability of the dengue vaccination among parents in urban poor communities of Quezon City, Philippines before and after vaccine suspension. BMC research notes, 11(1), 661. https://doi.org/10.1186/s13104-018-3766-y

Viennet, E., Ritchie, S. A., Williams, C. R., Faddy, H. M., \& Harley, D. (2016). Public Health Responses to and Challenges for the Control of Dengue Transmission in High-Income Countries: Four Case Studies. PLoS neglected tropical diseases, $10(9)$, e0004943. https://doi.org/10.1371/journal.pntd.0004943

Wang, W., Urbina, A., Chang, M., Assavalapsakul, W., Lu, P., Chen, Y., Wang, S. (2020, March 26). Dengue hemorrhagic fever - A systemic literature review of current perspectives on pathogenesis, prevention and control. Journal of Microbiology, Immunology and Infection. https://doi.org/10.1016/j.jmii.2020.03.007

World Health Organization (2009). Epidemiology, Burden of Disease and Transmission. Dengue: Guidelines for Diagnosis, Treatment, Prevention and Control: New Edition. Retrieved from https://www.ncbi.nlm.nih.gov/books/NBK143159/

World Health Organization (2020). Dengue and severe dengue. Retrieved September 26, 2020,from https://www.who.int/en/newsroom/fact-sheets/detail/dengue-and-severe-dengue

Yboa, B. \& Labrague, L. (2013). Dengue Knowledge and Preventive Practices among Rural Residents in Samar Province, Philippines American Journal of Public Health. 1. 47 - 52. 10.12691/ajphr-1-2-2. 